\title{
Analysis of the socio-economic development of the pilahuín parish, ambato canton, province of tungurahua during the years 2019 - 2021
}

\section{Análisis del desarrollo socioeconómico de la parroquia pilahuín, cantón ambato, provincia de tungurahua durante los años 2019 - 2021}

DOI: $10.46932 / \operatorname{sjdv} 3 n 1-097$

Received in: Jan 30st, 2021

Accepted in: Feb 1th, 2022

\author{
Fernando Fredi Rea García \\ Maestro en Gobierno y Gestión Local \\ Universidad Estatal de Bolívar \\ Av. Ernesto Che Guevara s/n y Av. Gabriel Secaira, Guaranda, Ecuador. \\ E-mail: frea@ueb.edu.ec \\ Taguada Nuñez Jazmin Alexandra \\ Licenciada en Sociologia \\ E-mail: jazmintaguada1603@gmail.com \\ Sheila Janet Rangel Gómez \\ Maestra en Gobierno y Gestión Local \\ Universidad Estatal de Bolívar \\ Av. Ernesto Che Guevara s/n y Av. Gabriel Secaira,Guaranda,Ecuador. \\ E-mail: srangel@ueb.edu.ec \\ José Luis Domínguez Caiza \\ Master en Gestión y liderazgo Educacional \\ Universidad Estatal de Bolívar \\ Av. Ernesto Che Guevara s/n y Av. Gabriel Secaira,Guaranda,Ecuador \\ E-mail: jdominguez@ueb.edu.ec
}

\section{RESUMEN}

El siguiente trabajo de investigación tiene por objetivo visualizar y analizar el desarrollo socioeconómico de la Parroquia Rural Pilahuín a partir de una perspectiva del Desarrollo Rural y Local, siendo este uno de los lugares económicamente más activos y con una población indígena que de acuerdo con los datos mostrados por El Heraldo (2018) "está conformada por 12.128 habitantes, según datos del último censo realizado en el 2010, de los cuales, el 90\% son indígenas y el 10\% son mestizos". (Párrafo 3) Sin embargo, dada la extensión de la población tanto en barrios y comunidades, se consideró primordial delimitar el estudio a la zona centro de la parroquia Pilahuín, al ser el lugar en el que se desarrollan las actividades comerciales y permite un muestreo aleatorio a partir de la población total de familias que son 569. En este sentido, es de suma importancia realizar investigación a una parroquia rural que representa un alto índice de pobreza a nivel nacional, por lo cual, es fundamental revisar el desarrollo de las condiciones de vida de sus habitantes y cómo la falta de políticas públicas e intervención gubernamental afecta el desarrollo social y económico de las familias, para ello, se empleó un método mixto en el que se realizó una recopilación bibliográfica-teórica del Desarrollo Rural y Local y posteriormente se analizaron e interpretaron los datos de una encuesta en la que se evidenciara el desarrollo socioeconómico de la zona centro de Pilahuín. 
Palabras clave: Desarrollo Socioeconómico, Desarrollo Local, Desarrollo Rural, Pilahuín.

\begin{abstract}
The following research work aims to visualize and analyze the socioeconomic development of the Rural Pilahuín Parish from a perspective of Rural and Local Development, this being one of the most economically active places and with an indigenous population that according to The data shown by El Heraldo (2018) "is made up of 12,128 inhabitants, according to data from the last census carried out in 2010, of which 90\% are indigenous and 10\% are mestizo." (Paragraph 3) However, given the extension of the population both in neighborhoods and communities, it was considered essential to delimit the study to the central area of the Pilahuín parish, as it is the place where commercial activities take place and allows a sampling random from the total population of families that are 569. In this sense, it is of the utmost importance to carry out research in a rural parish that represents a high rate of poverty at the national level, therefore, it is essential to review the development of the living conditions of its inhabitants and how the lack of public policies and Government intervention affects the social and economic development of families, for this, a mixed method was used in which a bibliographic-theoretical compilation of Rural and Local Development was made and later the data of a survey were analyzed and interpreted in which It will show the socioeconomic development of the central zone of Pilahuín.
\end{abstract}

Keywords: Socioeconomic Development, Local Development, Rural Development, Pilahuín.

\title{
1 INTRODUCCIÓN
}

El siguiente trabajo de investigación realiza un análisis socioeconómico de los habitantes de la zona centro de la parroquia Pilahuín, cantón Ambato, para conocer el estado actual de los mismos, en conjunto con una revisión teórica de dos líneas del desarrollo, las cuales son: el desarrollo rural y el desarrollo local manteniendo la perspectiva de estos dos modelos son propuestas novedosas que conforman una serie de elementos acordes a los territorios rurales y que en experiencias a nivel mundial han demostrado un impacto positivo al disminuir los índices de pobreza y desigualdad en estos sectores, recordando siempre que la meta de los Estados y gobiernos es llevar a la par el desarrollo económico y el desarrollo social.

El interés de realizar este trabajo de investigación es por la nula actualización y aplicación de un censo desde el año 2010, por lo que se desconoce la situación actual del país y de la parroquia Pilahuín que al ser uno de los territorios indígenas y ser considerado de alta producción agrícola, es de suma importancia que a través de datos estadísticos se puedan generar políticas públicas y planes de desarrollo, sean estos de corte social, político, económico o territorial que con datos solidos sustenten las acciones a emprender por los entes encargados.

Por lo tanto, con lo dicho con anterioridad la investigación es uno de los primeros estudios que persiguen analizar las condiciones socioeconómicas de los pobladores de la zona centro de la parroquia Pilahuín y que servirán de referencia para futuras investigaciones o formulaciones de planes de intervención dentro del sector a través de la detección de las necesidades primordiales de esta población. 


\subsection{LAS IMPLICACIONES SOCIALES DEL DESARROLLO RURAL}

En la actualidad en Latinoamérica, se ha venido desarrollando una temática importante para su análisis y discusión derivado de la importancia de las nuevas formas de organización, producción y formas de observar el comportamiento de las sociedades rurales y que a través de la investigación se busca la elaboración y aplicación de nuevos modelos de intervención por parte de los gobiernos nacionales y primordialmente localespara la eliminación de la pobreza rural, que aún prevalece en estos sectores. En tal sentido,el desarrollo rural se encuentra vinculado al campo y este al crecimiento económico de los países, por medio de la modernización de los sistemas de producción en la agriculturay la ganadería, así como el uso de insumos químicos que favorezcan el aumento de la productividad para satisfacer las necesidades de las sociedades en general. Pero ¿a qué sepuede definir como desarrollo?

\footnotetext{
El desarrollo es concebido como un proceso de cambio social, deliberado, cuyo objetivo último es la igualación de oportunidades sociales, políticas y económicas, tanto en el plano nacional como en las relaciones con otras sociedades más avanzadas, que coloca el acento en la acción, en los instrumentos de poder político y en las propias estructuras de poder para la orientación eficacia, intensidad y naturaleza del cambio. (Mujica y Rincón, 2010, p. 300)
}

Sin embargo, en el sector rural y su realidad se ha resignificado y evolucionado, y es así como se ha modificado su concepto, han tomado más importancia las actividades relacionadas a lo productivo agropecuario y también, las nuevas formas de generación de ingresos de las familias rurales, que han optado por otras formas organizacionales, como la cooperativa solo por mencionar un ejemplo. Entonces, he aquí un ejemplo de la nueva definición de desarrollo rural que no solo se ha convertido en una fuente importante de economía sino de administración, toma de decisiones, rescate de valores sociales y culturales, entre otros, por lo tanto, no solo se le debe de hacer sinónimo de campo o de agricultura. "El problema del desarrollo rural no puede pasar únicamente por acciones de apoyo a las actividades productivas, principalmente enfocadas en las familias pobres”. (Pachón, 2007, p. 52) Así, el concepto del desarrollo rural es:

En general, todo lo que no es "urbano" es "rural", de modo dicotómico, sin mayores subdivisiones de "rural". Solo algunos países (como Costa Rica y Brasil) introducen conceptos como "rural disperso", "rural concentrado" y "periferia urbana" o similares. La mayoría de las definiciones censales en uso en la región fueron diseñadas para la organización de la ronda de Censos de 1960, sin debate conceptual y sin modificaciones sustanciales desde entonces. (CEPAL, 2011, citado en Dirven, 2019, p. 6).

A su vez, también puede ser considerado como "el proceso de revitalización equilibrado y autosostenible del mundo rural basado en su potencial económico, social y medioambiental mediante una política regional y una aplicación integrada de medidas con base territorial por parte de organizaciones participativas". (Quintana, Cazorla y Merino, 1999, citados en Nogales, 2006, p. 9) 
Dentro de este contexto, es de gran preocupación que los gobiernos nacionales (Estados) no han prestado la atención requerida a los escenarios rurales ya que es el mercado su prioridad al ser considerado como el eje de la economía, de la vida social y política de los productores rurales. Es así, que el mercado se encuentra acorde con el modelo económico actual en su estado neoliberal y lo único que se busca es que la economía se ajuste de alguna manera para tratar de subsanar las condiciones socioeconómicas que cada vez se encuentran más deterioradas y son en las que vive la mayor parte de la población rural.

\subsection{EL MODELO DE DESARROLLO LOCAL COMO MOTOR DE LA RURALIDAD}

Como se mencionaba anteriormente, el desarrollo rural es un modelo para el abordaje de las reconfiguraciones de los sectores rurales, su forma de organización, producción, relación, y cohesión con el Estado, por medio de la implementación de políticas públicas que generen proyectos dirigidos principalmente al campo, es decir, a la agricultura a través de insumos químicos o maquinaria. Sin embargo, se destacaba que los sectores rurales no solo se caracterizan por ello, sino que actualmente son un reto para los Estados y gobiernos locales ya que la pobreza no ha disminuido a pesar de las intervenciones o generación de emprendimientos.

El desarrollo local es un tema de actualidad en América Latina, por lo que diversos investigadores, instituciones, gobiernos y estados lo nombran e incluyen como uno de los principales temas en sus planes o agendas. Las condiciones sociales y políticas actuales hacen que se piense en alternativas nuevas de desarrollo. Entre ellos se encuentra el desarrollo local aunado con la descentralización del Estado, para que los gobiernos locales tomen la autonomía en su toma de decisiones, sin embargo, estos aun dependen del impulso monetario del estado para la ejecución de los proyectos, pero, estas dos propuestas de desarrollo muestran tener potencial ya que representan una estrategia diferente para el desarrollo. De acuerdo con Cárdenas, 2012, es, además, "un proceso construido diferenciadamente, estructurador de nuevas formas de organización social, complejo, dinámico y multidimensional que implica procesos societales que van desde lo económico- productivo".

Aunado a esto, en el contexto latinoamericano se encuentra impactado por una de las herramientas del capitalismo que se le conoce como globalización, en conjunto, con constantes crisis sociales, económicas y políticas, además de reformas económicas de corte neoliberal que no han impactado de forma positiva en la reducción de la pobreza, ni ha mejorado las brechas de desigualdad, ni mucho menos se ha mejorado la calidad de vida de la población ecuatoriana. En la actualidad, tiene un aumento considerable de pobres, derivado de la pandemia, así como la reconfiguración de los espacios y de las relaciones sociales, políticas y económicas, por tal motivo, esta conlleva una rediscusión de los modelos de desarrollo implementados y los que se llevaran a cabo, sean estos nuevos o ya implementados, entre ellos, el del 
desarrollo local. Pero ¿qué puede entenderse como desarrollo local? En concordancia con Valenciano y Carretero, 2006:

\begin{abstract}
es el conjunto de iniciativas que tienen como objetivo hacer competitivas a las ciudades o territorios mediante el mejor aprovechamiento de sus recursos y factores de atracción. Combina las inversiones e iniciativas de los actores locales y los externos, impulsa la mejora del conocimiento y el aprendizaje de las organizaciones, la interacción entre los actores que toman las decisiones de intervención sobre el territorio y la sinergia entre las medidas. (p. 59)
\end{abstract}

Es así, que se considera de vital importancia que en el proceso de desarrollo intervengan cada uno de los actores económicos, sociales e institucionales del territorio, formando de esta manera un modelo de relaciones productivas, comerciales, tecnológicas, culturales e institucionales, cuya unión favorezcan los procesos de crecimiento, desarrollo y cambio. "El desarrollo de un sistema socio-político y económico se alcanza a partir del equilibrio entre cuatro dimensiones: institucional, social, económico y ambiental". [7. p 3] embargo, el único problema es que la cohesión de todos los actores, incluyendo los partidos políticos, los individuos, el sector privado, las instituciones públicas y el Estado se plantean diversas soluciones y no hay un consenso, pero, todas enfocadas hacia el desarrollo local y la participación de los actores son un común denominador.

\title{
1.3 EL DESARROLLO LOCAL Y RURAL COMO PROMOTORES DEL DESARROLLO
} SOCIOECONÓMICO

Hablar de desarrollo implica muchas formas de abordarlo, pero, en los países latinoamericanos se le utiliza como un grupo de pautas que generan un conjunto de mejoras, no solo en cuestiones económicas, sino que deben de ir de la mano con la evolución social, por lo tanto, al desarrollo se le puede definir desde el sentido social y el económico, como bien lo menciona Reyes, (2001):el término desarrollo se entiende como una condición social dentro de un país, en la cual las necesidades auténticas de su población se satisfacen con el uso racional y sostenible de recursos y sistemas naturales. La utilización de los recursos estaría basada en una tecnología que respeta losaspectos culturales y los derechos humanos. [...] En términos económicos, la definición mencionada anteriormente indica que para la población de un país hay oportunidades de empleo, satisfacción de por lo menos las necesidades básicas, y una tasa positiva de distribución y de redistribución de la riqueza nacional. (p. 1)

El desarrollo social y económico no es únicamente la creación de política socioeconómica y se ha demostrado que el crecimiento y la política económica no pueden asegurar objetivos sociales sin tomar en cuenta los contextos, sean estos nacionales, regionales, rurales o urbanos. Con esto, se trata de tener una visión integral del desarrollo que implica integrar tanto políticas sociales, económicas, ambientales y de ordenamiento democrático, entre capital humano, bienestar social, desarrollo sostenible y ciudadanía, de 
esta manera se podría afirmar que existe un verdadero desarrollo que se enfoque de forma multidimensional. En América Latina el pensamiento de lo local ha comenzado a tener énfasis en las propuestas del desarrollo y lo que denota en un agotamiento del Estado como el impulsor del desarrollo que trae como consecuencia constantes crisis en el sentido económico, político y social que pone en riesgo la democracia que es la meta del desarrollo. De esta manera surge el Desarrollo Local como formas de iniciativas en comunidades rurales y que nace como una alternativa a las diferentes crisis de los países, desde iniciativas de las personas en sus comunidades explotando sus capacidades en las diversas áreas.

En este contexto, en el que la economía se ha vuelto globalizada, en la que lo local se convierte en mundial y reivindica el uso de los recursos vistos como un conjunto de expresiones económicas, sociales, institucionales, culturales y políticas es necesario evaluar el desarrollo de territorios locales, para de este modo tratar de mitigar los efectos negativos de la globalización y el capitalismo. Entonces, el desarrollo rural es importante en el Desarrollo Socioeconómico al considerarse como "un sistema de intercambio abierto, globalizado, [con] la importancia que tiene la participación de las organizaciones comunitarias en la participación e implementación de estrategias de Desarrollo Económico local.” (Nicolande, 2013, p. 17). Pero también deben considerarse como políticas para derivar el desarrollo de la economía y de la sociedad de los países de forma equilibrada y sostenible.

Este constituye un sustento al desarrollo de la vida comunitaria y su potencial para aprovechar los recursos y talentos de cada uno de los miembros de la localidad, generar empleos y mejorar las condiciones de vida en conjunto con las autoridades competentes y crear estrategias que impulsen el desarrollo socioeconómico para todos los grupos sociales. Así que, se debe posibilitar el crecimiento y desarrollo rural - local a partir de la identificación de las habilidades y especialización productivas de los habitantes que permita una competitividad.

Además, se debe de identificar en cada localidad los núcleos económicos claves que necesitan consolidarse como fuentes de riqueza de los territorios y de igual manera con aquellos proyectos locales rurales que son de reciente creación, con el fin de impulsar o fortalecer actividades económicas que permitan generar fuentes de ingresos y trabajo para la población, todo esto, tendrá una repercusión positiva en los sectores más vulnerables que se encuentran en las zonas rurales y la disminución de la pobreza con mayores oportunidades sociales y económicas, siempre y cuando el Estado garantice el bienestar de la población por medio de políticas públicas y programas adecuados para cada contexto. psico-sociocultural, político, social, ambiental, territorial hasta lo económico- productivo". 


\section{METODOLOGÍA}

El presente trabajo de investigación se utiliza el método de carácter mixto, En este sentido el cualitativo es un método el cual permite la recopilación de datos bibliográficos, por lo que llega a ser empleado en diferentes disciplinas. "La investigación cualitativa busca adquirir información en profundidad para poder comprender el comportamiento humano y las razones que gobiernan tal comportamiento” (Cagliani, 2015, sección de ¿Qué es un método cualitativo?, párrafo 1) sección de ¿Qué es un método cualitativo?, párrafo 1). Por otro lado, el método Cuantitativo Se centra en los hechos o causas del fenómeno social, con escaso interés por los estados subjetivos del individuo. Este método utiliza el cuestionario, inventarios y análisis demográficos que producen números, los cuales pueden ser analizados estadísticamente para verificar, aprobar o rechazar las relaciones entre las variables definidas operacionalmente, además regularmente la presentación de resultados de estudios cuantitativos viene sustentada con tablas estadísticas, gráficas y un análisis numérico. (Rodríguez, 2010, p.32). El número total de la población residente en la zona centro de la parroquia Pilahuín son 569 familias de las cuales se aplicó un muestreo probabilístico aleatorio simple, el cual consiste en tener el número total de la población y en el que cada elemento tiene la misma probabilidad de ser seleccionado. De esta manera, la muestra es de 64 familias. Esta investigación se realizó una encuesta con el fin de analizar el desarrollo rural de la parroquia Pilahuín y su desarrollo socioeconómico vinculado a las familias habitantes de la zona centro para determinar sus condiciones de vida. La encuesta se puede conceptualizar como: "una investigación realizada sobre una muestra de sujetos representativa de un colectivo más amplio, utilizando procedimientos estandarizados de interrogación con intención de obtener mediciones cuantitativas de una gran variedad de características objetivas y subjetivas de la población"

\section{RESULTADOS}

Una de las partes más importantes para evidenciar el desarrollo socioeconómico de la parroquia Pilahuín es a partir de los datos recopilados mediante la aplicación de una encuesta a 64 familias de la zona céntrica de la parroquia. A continuación, se presentan los datos recopilados acompañados de un análisis de los datos más representativos en tablas de frecuencias y graficas de barras y circulares, entre ellas las mas importantes son: 
Tabla 1. Grupos de edad de los encuestados

\begin{tabular}{|c|c|c|c|c|}
\hline \multirow{2}{*}{ Detalle } & \multicolumn{2}{|c|}{ Frecuencia } & \multirow{2}{*}{ Total } & \multirow{2}{*}{ Porcentaje } \\
\cline { 2 - 3 } & Mujeres & Hombres & & \\
\hline De 15 a 19 años & 0 & 0 & 0 & 0 \\
\hline De 20 a 24 años & 2 & 0 & 2 & 3.13 \\
\hline De 25 a 29 años & 9 & 3 & 12 & 18.75 \\
\hline De 30 a 34 años & 1 & 0 & 5 & 1.56 \\
\hline De 35 a 39 años & 2 & 3 & 5 & 7.81 \\
\hline De 40 a 44 años & 3 & 2 & 6 & 9.81 \\
\hline De 45 a 49 años & 1 & 5 & 6 & 9.38 \\
\hline De 50 a 54 años & 1 & 5 & 9 & 14.06 \\
\hline De 55 a 59 años & 0 & 9 & & 5 \\
\hline
\end{tabular}

Nota: Elaboración propia con base en los datos obtenidos de la encuesta aplicada a 64 familias residentes en la zona centro de la Parroquia Pilahuín, Ambato, 2022.

Como se observa con anterioridad, la mayoría de las familias encuestadas y sus representantes, sean estos madres o padres de familia, se encuentran mayoritariamente entre el rango de edades de 25 a 29 años con un total de 18 personas que porcentualmente representan el $18.75 \%$, lo que significa que estas personas han comenzado sus familias a temprana edad. Sin embargo, en un segundo lugar, con una representación igualitaria del 14.06\%, correspondiente a dos rangos de edad, los cuales se comprenden de 55 a 59 años y de 65 a 69 años, proporcionan una interpretación interesante al entenderse que la población habitante de la zona centro de Pilahuín tiene un alto porcentaje de personas adultas.

Tabla 2. Grupo étnico de pertenencia

\begin{tabular}{|c|c|c|c|c|c|}
\hline Detalle & \multicolumn{2}{|c|}{ Frecuencia } & Total & Porcentaje \\
\hline & Hombres & Mujeres & & \\
\hline Indígena & 18 & & 19 & 37 & 57.81 \\
\hline Mulato/a & 0 & & 0 & 0 & 0.00 \\
\hline Montubio/a & 0 & & 0 & 0 & 0.00 \\
\hline Mestizo/a & 16 & & 10 & 26 & 40.63 \\
\hline Blanco/a & 0 & & 0 & 0 & 0.00 \\
\hline Otro/a & 1 & & 0 & 1 & 1.56 \\
\hline Total & 35 & & 29 & 64 & 100 \\
\hline
\end{tabular}

Nota: Elaboración propia con base en los datos obtenidos de la encuesta aplicada a 64 familias residentesen la zona centro de la Parroquia Pilahuín, Ambato, 2022.

La tabla 2, en concordancia con la tabla 3, muestra que, de las 64 personas encuestadas, 37 de ellas se identifican como indígenas, representando un porcentaje del $57.81 \%$, de los cuales, en su mayoría son mujeres; todo ello, corresponde al censo de población 2010 que identifica a la mayoría de la población de esta parroquia como indígena. En segundo lugar, con 26 personas que forman parte del $40.63 \%$ se auto determinan como mestizos, siendo en su mayoría del sexo masculino, lo cual es importante al tratarse de una cuestión probable de perdida de usos, costumbres y prácticas que históricamente se llevaban a cabo en este sector, en conjunto con las relaciones socio afectivas con personas pertenecientes a otros cantones 
Tabla 3. Porcentaje de migración

\begin{tabular}{|l|l|l|l|l|}
\hline \multirow{2}{*}{ Detalle } & \multicolumn{2}{l|}{ Frecuencia } & Total & Porcentaje \\
\hline & Hombres & Mujeres & & \\
\hline No ha migrado & 33 & 29 & 62 & 96.88 \\
\hline Argentina & 0 & 0 & 0 & 0.00 \\
\hline España & 1 & 0 & 1 & 1.56 \\
\hline Italia & 0 & 0 & 0 & 0.00 \\
\hline Cuba & 0 & 0 & 0 & 0.00 \\
\hline Estados Unidos & 0 & 0 & 0 & 0.00 \\
\hline Israel & 0 & 0 & 0 & 0.00 \\
\hline Venezuela & 0 & 0 & 0 & 0.00 \\
\hline Sin especificar & 1 & 0 & 1 & 1.56 \\
\hline Total & 35 & 29 & 64 & 100 \\
\hline
\end{tabular}

Nota: Elaboración propia con base en los datos obtenidos de la encuesta aplicada a 64 familias residentes en la zona centro de la Parroquia Pilahuín, Ambato, 2022.

En lo referente a la migración hacia otros países, es claramente explicito que 62 personas habitantes de la zona centro de la parroquia Pilahuín porcentualmente representadas con el $96.88 \%$ no han migrado, pero se registra que hay personas que habían migrado, pero ya han retornado al país. Además, solo 1 persona, equivalente al $1.56 \%$ ha migrado a España y otra no ha especificado el lugar. Como dato extra, en el censo 2010, se observa que la parroquia registraba en ese año un $71.88 \%$ de migración hacia España, seguido de Venezuela con $9.38 \%$ y otros países con el mismo porcentaje, es así, que se evidencia su disminución por lo menos en la zona céntrica.

Tabla 4. Atención y asistencia a establecimientos de salud

\begin{tabular}{|l|l|l|l|l|}
\hline Detalle & Frecuencia & Total & Porcentaje \\
\cline { 2 - 2 } & & & & \\
\cline { 2 - 3 } & Hombres & Mujeres & & \\
\hline
\end{tabular}

\begin{tabular}{|l|l|l|l|l|}
\hline Ministerio de Salud & 24 & 16 & 40 & 62.50 \\
\hline Establecimiento del IESS & 9 & 1 & 10 & 15.63 \\
\hline Hospital Clínica Particular & 1 & 9 & 10 & 15.63 \\
\hline Otro & 1 & 3 & 4 & 6.25 \\
\hline Total & 35 & 29 & 64 & 100 \\
\hline
\end{tabular}

Nota: Elaboración propia con base en los datos obtenidos de la encuesta aplicada a 64 familias residentes en la zona centro de la Parroquia Pilahuín, Ambato, 2022.

Uno de los problemas que mencionaron los encuestados es que seguía perdurando en la parroquia el poco acceso a medicamentos básicos y a atención a problemas de salud más específicos, sin embargo, este es el caso particular de los sectores rurales a nivel nacional, aun así, cuando la población requiere de 
atención médica el $62.50 \%$ correspondiente a 40 personas asiste al Ministerio de Salud, seguidamente y a la par con el $15.63 \%$ las demás personas afirmaron que asisten al Instituto Ecuatoriano de Seguridad Social (IESS) y al hospital/clínica particular. Y un dato a resaltar es que el 6\% dijo asistir a otro tipo de establecimientos que se dedican a la curación de enfermedades con medicina tradicional, con chamanes o con atención en casa, recordando siempre que la tradición de las comunidades y pueblos indígenas es atender y dar seguimiento de enfermedades de forma diferente y solo cuando realmente es necesario asisten a los hospitales

Tabla 5. Nivel de instrucción

\begin{tabular}{|c|c|c|c|c|}
\hline \multirow{3}{*}{ Detalle } & \multirow{2}{*}{\multicolumn{2}{|c|}{ Frecuencia }} & \multirow{3}{*}{ Total } & \multirow{3}{*}{ Porcentaje } \\
\hline & & & & \\
\hline & Hombres & Mujeres & & \\
\hline Alfabetización & 1 & 3 & 4 & 6.25 \\
\hline Educación Básica & 20 & 15 & 35 & 54.69 \\
\hline Educación Media & 8 & 4 & 12 & 18.75 \\
\hline Superior & 2 & 2 & 4 & 6.25 \\
\hline Posgrado & 0 & 0 & 0 & 0 \\
\hline Desconoce & 4 & 5 & 9 & 14.06 \\
\hline Total & 35 & 29 & 64 & 100 \\
\hline
\end{tabular}

Nota: Elaboración propia con base en los datos obtenidos de la encuesta aplicada a 64 familias residentes en la zona centro de la Parroquia Pilahuín, Ambato, 2022.

Como bien se mencionó con anterioridad, la mayoría de los padres y madres de familia encuestados se encuentran en un rango de edad joven, lo cual, ha incidido de forma paulatina en la parroquia, puesto que al conformar familias a temprana edad repercute en el nivel de instrucción ya que tuvieron que dedicarse a trabajar en lugar de estudiar paramantener a la familia u otra es porque no se cuenta con el suficiente dinero para seguir estudiando y es de vital importancia ayudar a los padres en el campo, por lo cual se puede observar, que la mayoría con un 54.69\% referente a 35 personas indicaron que solo pudieron acceder a la educación básica. Después, el 18.75\% ha cursado hasta la educación media, de ahí el $6.25 \%$, únicamente 4 personas han podido acceder a la educación superior, lo que se presta a la interpretación de que aún no se ha impulsado de alguna manera que los jóvenes sigan estudiando o que no se ha generado algún tipo de política pública que permita disminuir estas cifras. 
Figura 1. Actividad económica por rama de actividad

\begin{tabular}{|c|c|}
\hline Otros & 0.00 \\
\hline No trabaja & 20.31 \\
\hline $\begin{array}{c}\text { Actividades de los hogares como } \\
\text { empleadores }\end{array}$ & 1.56 \\
\hline $\begin{array}{c}\text { Otras actividades comunitarias sociales y } \\
\text { personales de tipo servicios }\end{array}$ & 6.25 \\
\hline Artes, entretenimiento y recreación & 0.00 \\
\hline $\begin{array}{c}\text { Actividades de servicios administrativos y } \\
\text { de apoyo }\end{array}$ & 0.00 \\
\hline Actividades de servicios sociales y de salud & 3.13 \\
\hline $\begin{array}{c}\text { Actividades profesionales, científicas y } \\
\text { técnicas }\end{array}$ & 0.00 \\
\hline Enseñanza & 1.56 \\
\hline Administración pública y defensa & 1.56 \\
\hline $\begin{array}{c}\text { Actividades inmobiliarias, empresariales y } \\
\text { de alquiler }\end{array}$ & 0.00 \\
\hline Información y comunicación & 0.00 \\
\hline Intermediación financiera & 4.69 \\
\hline $\begin{array}{c}\text { Transporte, almacenamiento y } \\
\text { comunicaciones }\end{array}$ & 1.56 \\
\hline Hoteles y restaurantes & 0.00 \\
\hline Comercio al por mayor y al por menor & 9.38 \\
\hline Construcción & 6.25 \\
\hline $\begin{array}{c}\text { Distribución de agua, alcantarillado y } \\
\text { gestión de deshechos }\end{array}$ & 0.00 \\
\hline Suministros de electricidad, gas y agua & 0.00 \\
\hline Industrias manufactureras & 3.13 \\
\hline Explotación de minas y canteras & 10.94 \\
\hline Agricultura, ganadería, caza y silvicultura & \\
\hline
\end{tabular}

Nota: Elaboración propia con base en los datos obtenidos de la encuesta aplicada a 64 familias residentes en la zona centro de la Parroquia Pilahuín, Ambato, 2022.

La agricultura es la ocupación principal de los hombres y mujeres de la parroquia Pilahuín, zona centro, con el $29.69 \%$, seguido de un $20.31 \%$ de encuestados que no realizan actividades asalariadas, 
además, ambos sexos tienen trabajos diversos que van desde la construcción (6.25\%), hasta la intermediación financiera (4.69\%), al comercio al por mayor y al por menor (9.38\%) y a otras actividades comunitarias sociales y personales de tipo servicios $(6.25 \%)$.

Pero, el trabajo considerado más pesado como es la explotación de minas y canteras con un 10.94\% o la construcción con un $6.25 \%$ es un trabajo destinado al sexo masculino, no solo socialmente hablando sino demostrado en esta encuesta; mientras que las mujeres se dedican a actividades de servicios sociales y de salud (3.13\%), a la enseñanza (1.56\%) y mayoritariamente no trabajan, pero en los hogares se dedican a otras actividades que cabe recalcar no son remuneradas ni consideradas como trabajo pero en la actualidad a través de la teoría feminista y sus estudios se ha demostrado que este si es un trabajo y muchas ocasiones es llamado trabajo social.

Tabla 4. Ingreso mensual de las personas económicamente activas

\begin{tabular}{|l|l|l|l|l|}
\hline & Frecuencia & & \\
\hline Detalle & & & Total & Porcentaje \\
\hline & Hombres & Mujeres & & \\
\hline$\$ 100-\$ 300$ & 15 & 21 & 36 & 56.25 \\
\hline$\$ 300-\$ 600$ & 15 & 5 & 20 & 31.25 \\
\hline$\$ 600-\$ 900$ & 5 & 3 & 8 & 12.5 \\
\hline$\$ 900-\$ 1000-$ o mas & 0 & 0 & 0 & 0 \\
\hline Total & 35 & 29 & 64 & 100 \\
\hline
\end{tabular}

Nota: Elaboración propia con base en los datos obtenidos de la encuesta aplicada a 64 familias residentes en la zona centro de la Parroquia Pilahuín, Ambato, 2022.

Como ya se denoto, la principal actividad de los habitantes de la zona centro de la parroquia Pilahuín se dedica a la agricultura, lo cual no representa un ingreso fijo como lo mencionaron algunos de los encuestados, por lo tanto, el ingreso de las familias de forma mensual en su mayoría representando con el 56.25\% asciende entre $\$ 100$ y $\$ 300$ dólares mensuales, que no cubren en su totalidad las necesidades de las familias pilahueñas, en segundo lugar con el $31.25 \%$ se encuentran familias que perciben entre $\$ 300$ y $\$ 600$ dólares. En tercer lugar, con el $12.5 \%$ o sea solo 8 familias aseveran percibir entre \$600 y \$900 dólares mensuales y finalmente, ninguno de los habitantes de esta zona supera los \$900 dólares que puede indicar el bajo nivel de instrucción, la actividad económica a la que se dedican entre otros factores. Cabe agregar que de acuerdo con el Índice de Precios en el Ecuador (2020) sobre la compra de una canasta básica familiar en la zona sierra en la que se encuentran los productos básicos tiene un costo de $\$ 730.98$ y la canasta vital que tiene un precio de $\$ 508.52$, esto indica que estas familias no cubren sus necesidades básicas. 
Tabla 5. Tipo de vivienda

\begin{tabular}{|c|c|c|c|c|}
\hline \multirow{3}{*}{ Detalle } & \multirow{2}{*}{\multicolumn{2}{|c|}{ Frecuencia }} & \multirow{3}{*}{ Total } & \multirow{3}{*}{ Porcentaje } \\
\hline & & & & \\
\hline & Hombres & Mujeres & & \\
\hline Casa o villa & 34 & 27 & 61 & 95.31 \\
\hline Departamento & 0 & 0 & 0 & 0.00 \\
\hline Cuarto & 1 & 2 & 3 & 4.69 \\
\hline Mediagua & 0 & 0 & 0 & 0.00 \\
\hline Rancho & 0 & 0 & 0 & 0.00 \\
\hline Covacha & 0 & 0 & 0 & 0.00 \\
\hline Choza & 0 & 0 & 0 & 0.00 \\
\hline Otra & 0 & 0 & 0 & 0.00 \\
\hline Total & 35 & 29 & 64 & 100 \\
\hline
\end{tabular}

Nota: Elaboración propia con base en los datos obtenidos de la encuesta aplicada a 64 familias residentes en la zona centro de la Parroquia Pilahuín, Ambato, 2022.

Con respecto al tipo de vivienda según el gráfico 15, se puede interpretar de la siguiente manera: la mayoría de las familias residentes en la zona centro habitan en una casa o villa con un $95.31 \%$ en la cual se puede apreciar un incremento desde el 2010 que demostraba un 71\%, pero aun el $4.69 \%$ se encuentra viviendo en cuarto ya según lo que mencionan las condiciones económicas no les han permitido mejorar y más ahora con la pandemia de covid-19 se han agravado algunas situaciones.

\section{DISCUSIÓN}

La zona centro de la parroquia Pilahuín y su desarrollo socioeconómico ha evidenciado un crecimiento y mejora positiva en algunas de las áreas débiles que surgieron en el censo del 2010, sin embargo, aún hay áreas a las que se debe de prestar mayor atención para mejorar las condiciones de vida de los habitantes ya que se ha priorizado más en la creación de infraestructura vial. A continuación, se mencionan las conclusiones o hallazgos más significativos de esta investigación.

En primer lugar, se debe de tener en cuenta que la organización social, política, económica y cultural de los habitantes está muy arraigada y muchas veces lo que para algunos significa desarrollo para otros no lo es como bien lo percibe el Estado, pero este mismo y los gobiernos sean de corte provincial o local deben de aceptar todo ello y crear verdaderas formas de impulsar los sectores rurales y no solo dejar en teoría el desarrollo local y rural que además de preservar las costumbres y tradiciones de los pueblos indígenas también impulsen su economía. Pero, aun sigue siendo un reto para el Ecuador y países hermanos llevar a la par el desarrollo social y el desarrollo económico en especial de estos sectores rurales.

Hablando en términos demográficos, se ha identificado que la zona centro se encuentra principalmente habitada por hombres, de ahí que tanto hombres como mujeres se encuentran en edad adulta que va desde los 55 a los 70 años, pero que también existe una población joven de entre 25 a 30 años que se encuentran en edad reproductiva, lo que indica que han conformado familias a temprana edad pero que han decidido no incrementar el número de hijos por lo que se demuestra en la tabla 4. Aunado a 
este apartado, las personas en su generalidad se reconocen como indígenas en su mayoría mujeres a pesar de que va en aumento el número de personas que se identifican como mestizas.

En lo referente a migración y emigración se denota que no hay mucha emigración sobre todo de las mujeres que han impulsado otro tipo de trabajos para mantener a su familia y si bien, los hombres emigran hacia la ciudad más cercana como lo es Ambato, seguido de Quito y que se da a una reflexión de que trabajo hay en la parroquia, pero se encuentra en el campo.

En cuanto a la salud, existe una mayor preocupación por atenderse de mejor manera, pero que no requiera mucho dinero y también como se pudo evidenciar la mayoría de la población no cuenta con algún tipo de servicio médico pagado que pueda cubrir sus necesidades médicas inmediatas, por lo cual, la población asiste al Ministerio de Salud, no obstante, este establecimiento no se encuentra equipado en su totalidad y la falta de medicinas y atención a algunas enfermedades es nula. En cuanto a la preferencia por el IESS o la clínica privada estas se encuentran a la par, pero con mayor frecuencia las mujeres prefieren asistir a la clínica privada. También, aunque es significativo, la población prefiere curarse en casa, con medicina tradicional o visitando a chamanes sobre todo es una práctica que realizan las personas mayores. En conjunto con esto, se aprecia que a pesar de la pandemia de covid-19, la zona centro de Pilahuín no ha registrado altas tasas de mortalidad.

La educación de la parroquia se le puede denominar crítica, ya que muchos de los pobladores han solo llegado a la Educación Básica y han adquirido la habilidad de leer y escribir, esto debido al poco interés de la población joven que se interese en estudiar, además de que no ha mejorado la calidad educativa y que para seguir estudiando y obtener el grado se debe de abandonar el hogar y dirigirse a la ciudad más pronta, todo ello ha generado que las personas prefieran dedicarse a la fuente principal de ingresos que es a la producción agrícola, teniendo como evidencia que únicamente 4 personas han podido culminar una carrera universitaria.

La economía de la gran mayoría de la población de la zona depende de la producción agrícola, en su mayoría, tanto hombres y mujeres trabajan y se dedican a la misma actividad que es la agricultura y ganadería, siendo entonces el comercio una de las formas de obtener ingresos económicos. Seguido de la agricultura, los habitantes se dedican a diferentes actividades económicas como bien se pudo observar en la tabla 13, sin embargo, las mujeres denotan en alto porcentaje que no realizan alguna actividad económica. Al ser la agricultura la principal fuente de ingresos no existe de una forma equilibrada o fija de forma mensual, por lo que los habitantes coincidieron en que sus ingresos ascienden a los \$100 y \$300 dólares mensuales.

Respecto con la vivienda y los servicios básicos de los habitantes, se mostró que las familias habitan en casas o villas, que en su mayoría son propias, a excepción de un pequeño porcentaje que renta 
o vive con familiares cercanos. Además, las casas están hechas de hormigón como un material básico pero que con las condiciones climáticas de la zona no resguardan el calor, seguido del ladrillo o bloque, mientras que el adobe ya no es muy utilizado. En cuanto a los servicios, correspondientes al abastecimiento de agua, electricidad y eliminación de desechos sólidos, los dos primeros tienen una cobertura completa, ambos conectados a la red pública abasteciendo a la población y el tercero, aunque la mayoría de los encuestados afirmaron utilizar el carro recolector aún se preservan las prácticas de arrojar a un terreno baldío o quebrada la basura o quemarla. Entonces, no existe alguna clase de seguridad referente al cuidado de los recursos naturales, protección de cultivos de mal clima o desastres naturales, ninguna clase de prevención y cuidado de la salud familiar y comunitaria.

\section{CONCLUSIONES}

Con todo lo recopilado se recomienda una mejor organización de los habitantes de la zona y de la Junta Parroquial para que se pueda impulsar proyectos y acciones que beneficien a la población mejorando su condición socioeconómica. Por ejemplo, que se desarrollen proyectos sociales, económicos y ambientales para generar fuentes de empleo y se concientice a los habitantes sobre las problemáticas comunitarias encontradas, así como promover la búsqueda de apoyos financieros para los proyectos de desarrollo social.

Los datos arrojados en la encuesta sugieren la creación de una organización agropecuaria comunitaria, la cual pueda impulsar capacitaciones sobre técnicas de cultivo que permitan incrementar la producción y a su vez regule las prácticas comerciales y promueva la siembra de productos diversos que generen una competencia equilibrada.

Hacer un llamado a las instituciones públicas, sean estas de salud, educación, o políticas que incidan a través de la creación de verdaderas políticas públicas que vinculen a los habitantes, a los entes políticos llámense parroquiales, provinciales y nacionales para tener un impacto positivo en las familias residentes de la parroquia y se vean mejoradas sus condiciones de vida. Finalmente, que se analicen nuevas propuestas de desarrollo que sean acordes a la zona, por ejemplo, el desarrollo local y rural que a través de otras experiencias en el mundo se ha demostrado su efectividad. 


\section{REFERENCIAS}

Cagliani, M. (2015). Tendenzias.com. Recuperado el 04 de noviembre del 2020 de: https://tendenzias.com/ciencia/que-es-el-metodo-cualitativo/

Dirven, M. (2019). Nueva definición de lo rural en América Latina y el Caribe en el marco de FAO para una reflexión colectiva para definir líneas de acción para llegar al 2030 con un ámbito rural distinto. Organización de las Naciones Unidas para la Agricultura y la Alimentación. http://www.fao.org/3/c5509es/ca5509es.pdf

El Heraldo, (2018, 29 de mayo). Pilahuín con historia y cultura. Recuperado el 07 de diciembre del 2020 de: https://www.elheraldo.com.ec/pilahuin-con-historia-y-cultura/

Mujica, N. y Rincón, S. (2010). El concepto de desarrollo: posiciones teóricas más relevantes. Revista Venezolana de Gerencia. vol. 15, (50). 294-320. https://www.redalyc.org/pdf/290/29015906007.pdf

Nicolalde, M. (2013). Plan de Desarrollo Local de la Parroquia Pilahuín del Cantón Ambato en la Provincia de Tungurahua. [tesis para la obtención de ingeniería, Universidad Central del Ecuador]. Repositorio Digital de la Universidad Central del Ecuador. http://www.dspace.uce.edu.ec/handle/25000/1700

Nicolalde, M. (2013). Plan de Desarrollo Local de la Parroquia Pilahuín del Cantón Ambato en la Provincia de Tungurahua. [tesis para la obtención de ingeniería, Universidad Central del Ecuador]. Repositorio Digital de la Universidad Central del Ecuador. http://www.dspace.uce.edu.ec/handle/25000/1700

Nicolalde, M. (2013). Plan de Desarrollo Local de la Parroquia Pilahuín del Cantón Ambato en la Provincia de Tungurahua. [tesis para la obtención de ingeniería, Universidad Central del Ecuador]. Repositorio Digital de la Universidad Central del Ecuador. http://www.dspace.uce.edu.ec/handle/25000/1700

Nogales, M. A. (2006). Desarrollo rural y desarrollo sostenible. La sostenibilidad ética. CIRIEC-España, Revista de Economía Pública, Social y Cooperativa. Núm. 55. Págs. 7-42. https://www.redalyc.org/pdf/174/17405502.pdf

Pachón, F. (2007). Desarrollo Rural: Más que Desarrollo Agrícola. Revista de la Facultadde Medicina $\begin{array}{lllllll}\text { Veterinaria } y & \text { de } & \text { Zootecnia. } & \text { vol. } & \text { 54, } & \text { (1). } & \text { Págs. }\end{array}$ https://www.redalyc.org/pdf/4076/407642324008.pdf.

Reyes, G. (2001). Principales Teorías sobre el Desarrollo Económico y Social. Revista Nómadas.

Rodríguez, M. (2010). Métodos de investigación. Ed. Universidad Autónoma de Sinaloa.

Valenciano, J. y Carretero, A. (2006). Desarrollo local en el mundo rural: El caso europeo. Perspectivas. Vol. 9, (3). Págs. $57-80$. 